\title{
Spacesuit Dust Removal Techniques in Microgravity
}

\author{
Aaron Olson, Julie Mason, Collin Bezrouk \\ UW-Madison 2012 Zero Team \\ University of Wisconsin- Madison
}

\begin{abstract}
Preventing and removing the build-up of regolith on an astronaut's space suit is a major issue facing future manned exploration missions. The dust poses a health threat to astronauts; it also adversely affects their equipment. The Field Integrated Regolith Cleaning Experiment (FIRCE) was designed to study different techniques for removing regolith as well as reviewing what commercial off-the-shelf products were best suited for cleaning space suit orthofabric and polycarbonate (used on the astronaut's visor). All of the commercial products worked well in both the 1-g environment as well as the 0 -g environment. All were ranked above a 7.0/10 for ease of use and above 7.6/10 for cleaning effectiveness. A custom made magnetic brush, designed to attract the regolith's static charge was not effective at removing dust because the humidity in the air prevented the build-up of static charge on the regolith.
\end{abstract}

\section{Introduction}

There are many design challenges in the area of manned space exploration, one that is very important, but may not be the first to come to mind is the regolith (dust) contamination challenge. The Field Integrated Regolith Cleaning Experiment (FIRCE), on a level one basis, addresses the question of which commercial off-the-shelf products could be used to mitigate the dust challenge.

\section{Background}

Dust mitigation has been identified as a major issue for future space exploration missions. Based on the experiences from the Apollo missions, it is evident that that dust contamination can cause mechanical and wear issues with the space suits as well as the critical components within the vehicle. Particles less than three microns in size can lodge in the lungs if inhaled. The nanophase iron in lunar dust can enter the bloodstream and potentially reach toxic levels ${ }^{[2]}$. The space suit is the single greatest transport mechanism for Extra-Vehicular Activities (EVA). Since a single suit system is utilized, a suit worn for an EVA must also be worn inside the vehicle for activities such as takeoff and landing. Not only will the suit need to be cleaned before re-entering the vehicle, the suit will also have to be cleaned before an EVA; organic materials from Earth will need to be contained in order to maintain the cleanliness of the exploration area ${ }^{[1]}$.

The main issue with space dust stems from its strange properties. The dust is formed from micrometeorite impacts pulverizing local rocks into fine particles. Energy from the collisions causes the dirt to melt into a vapor that forms a glassy shell when cooled. These micron sized angular particles are extremely abrasive and can wear through space suits as well as disable the joint mobility of the space suits. The irregular shapes of the dust particles and electrostatic cling causes the dust to adhere to exposed surfaces. The electrostatic cling is cause by the electron bombardment from solar winds charging the specs of metallic iron $(\mathrm{Fe} 0)$ which are embedded with the glassy shell of each particle ${ }^{[2]}$. 
The inherent nature of the dust particles to be electrostatically charged can potentially be utilized to mitigate dust contamination. By introducing a magnetic field, the charged dust particles can be removed from a contaminated material surface.

\section{Description of experiment}

The Field Integrated Regolith Cleaning Experiment (FIRCE) was designed as a level one test to determine effective techniques and products for removal of lunar regolith. This experiment investigated methods for dust removal on space suit materials in microgravity. The two tested materials were orthofabric and polycarbonate, which are used for the suit and visor of an EVA suit. Each flight performed testing on 5, 4 x 4 inch samples of orthofabric and 6, $1.5 \mathrm{x}$ 1.5 inch polycarbonate samples. The space suit materials were exposed to JSC-1A $<1 \mathrm{~mm}$ lunar dust simulant and mounted in a doubly contained glove box. During the flight, various dust removal techniques were performed on each of the samples, and the before-and-after effects of cleaning the samples were recorded via a video camera that was mounted to the outside of the box. Figure 1 shows the experiment setup.

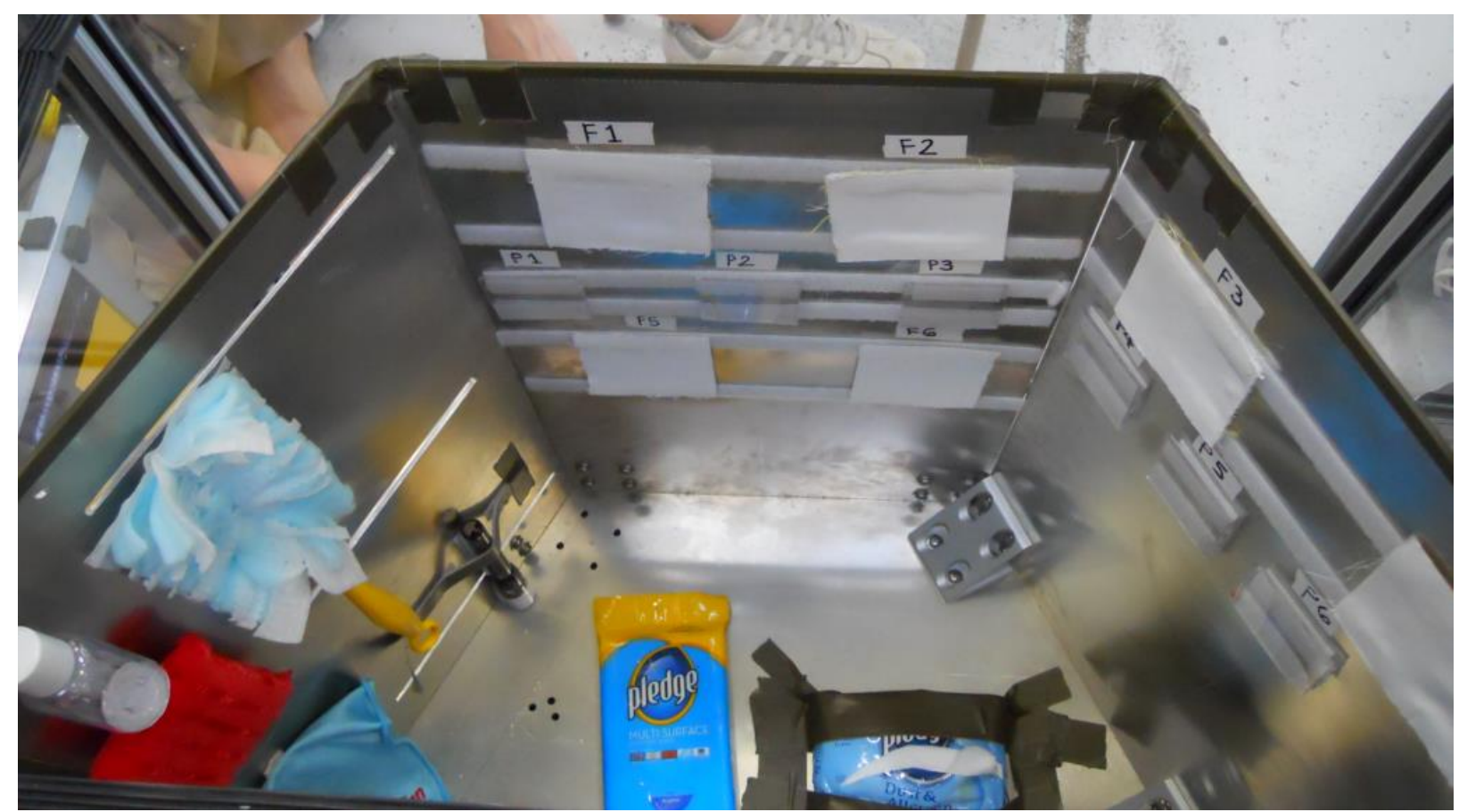

Figure 1: Isometric view of experiment setup inside glovebox

\section{Methods}

We began our research by following two paths to cleaning regolith from space suits: commercial products and magnetic dust removal. We intended to test both elements in order to better focus later research. On the magnetic side, we reviewed the magnetic properties of regolith, finding that most dust particles contain a core of ferrous iron. Additionally, the insulating glass coating on the dust is ideal for storing static charge. Commercial product research looked into different dust attraction mechanisms used in cleaning products as well as how each one prevents damage to the surface it is cleaning. A list of products suitable for safely cleaning electronic and camera equipment was put together for testing. 
Prior to the experiment we anticipated the commercial cleaning products would be close to $50 \%$ effective at removing dust. More specifically, they would be able to remove dust sitting on top of the sample, but due to the jagged glass edges on the dust particles, we hypothesized that it would be difficult to remove embedded particles. Since the commercial products rely on contact pressure for cleaning, we felt that many particles that were not originally embedded would become embedded during cleaning. The magnetic brush was also expected to remove about $50 \%$ of the lunar simulant from the samples. While it was a non-contact cleaning method, the strength of the magnetic field was not such that we felt it would be able to lift out embedded particles.

To give ourselves a better idea of how the cleaning products would respond in microgravity, we tested two orthofabric samples in 1-g with a horizontal configuration. One sample had lunar simulant dusted on its surface while the second sample had the dust rubbed into it. We were surprised to find that the Swiffer duster was able to remove almost all traces of the dust after applying very modest contact pressure. The magnetic brush was also tested under these conditions and was unable to lift any traceable amounts of dust from the samples. These results varied from our original hypothesis. We concluded that the high humidity in the air was causing a reduction of static charge, reducing the effectiveness of the magnetic brush. Conversely, the commercial cleaning products were substantially more effective. The experiment contained all dust, samples, and cleaning products in a doubly contained glove box with two portholes and gloves for the experimenter to run the test. The orthofabric and polycarbonate samples were mounted vertically on a wall inside the glove box. They had been pre-coated with JSC-1A lunar simulant, which had been dusted on and then rubbed into the sample in 1-g. Each cleaning product was mounted to the floor of the glove box or one of the vertical walls using Velcro. Only one item was tested at a time and all other cleaning items remained stowed during this process. We also mounted a small bottle filled with lunar simulant. This was applied to the samples between cleanings after all samples had been tested at least once. Regolith was applied to the sample and rubbed in before cleaning resumed. To test each sample, the cleaning tool was used as directed by the manufacturer. The cleaning involved a repeated scrubbing motion over the surface of one sample for a period of approximately 10 seconds. The sample was inspected for cleanliness and then the scrubbing was repeated with more pressure applied to the sample. When the experimenter felt that the sample had been thoroughly cleaned, he or she proceeded to the next sample and/or test article. Whenever a test item was finished, it was stowed using Velcro on the bottom of the containment box.

The magnetic brush was tested over five samples: two orthofabric, two polycarbonate, and once with free-floating regolith. The first orthofabric and polycarbonate samples were tested by turning on the magnetic brush, and passing it about 0.5 inches above the surface of the sample, being careful not to touch it. After approximately 10 seconds of waving, the brush head was inspected to see the dust it had collected. Any present dust was wiped away with the gloves and the test repeated two more times. The second orthofabric and polycarbonate samples were tested by pressing and scrubbing the magnetic brush over the sample.

Similarly, after about 10 seconds of rubbing, the head of the brush was inspected for dust, cleaned, and repeated. The results of these tests were poor, so we tested the brush on freefloating regolith in the containment box. Regolith was released into the air and the brush was 
waved about 1 inch away. The dust was attracted to the brush head in greater quantities than when trying to pull dust from the mounted samples.

\section{Results}

In the zero gravity testing conditions, most of the cleaning elements performed as expected and similarly to the $1 \mathrm{~g}$ results; the only exception was the magnetic brush. Since the experiment was conducted within the atmosphere, meaning it was subject to the effects of atmospheric pressure and humidity; the results were mitigated compared to the hypothesized results of the space environment. The humidity reduced the electrostatic charge of the dust to a level that rendered the magnetic brush useless.

\section{Data Collection}

Team members were asked to rate each item post flight in regards to effectiveness and ease of use for both cleaning of the polycarbonate and orthofabric materials. Each flyer was also asked to include comments about the usage of each item to potentially clean a large item such as a full space suit or a large solar panel; further testing is required to clarify the results gathered as well as test items in a vacuum or space simulation environment.

\section{Microgravity Results}

\section{Swiffer Duster (SD)}

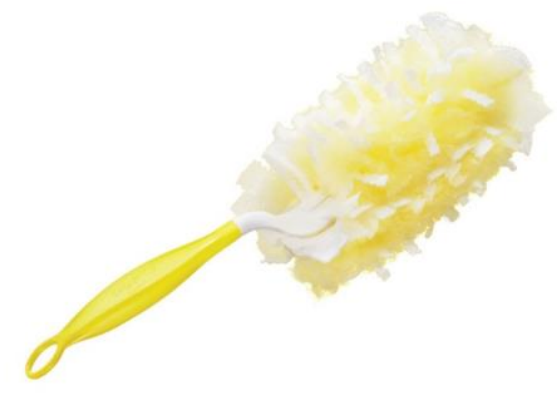

Figure 2: Swiffer Duster

The SD received a rating of $8.6 / 10$ for ease of use due to the ergonomic design of the handle and extension of flexible bristles on the end. Effectiveness averaged 8.6/10 with the polycarbonate and a 7.6/10 with the orthofabric. The SD proved generally more effective with the polycarbonate; owing to the flexibility of the bristles, it is difficult to apply pressure with this brush particularly for particles engrained in the

orthofabric. Overall, it seemed effective at cleaning by visual inspection and perhaps the ability to scrub would engrain particles deeper within the fabric. Furthermore the prediction that the bristles would not capture all dust but rather allow some to be re-emitted into the atmosphere proved to be incorrect. This was probably due to the large amount of bristles and small sample sizes. The SD should be further tested with larger samples.

\section{Microfiber Cloth}

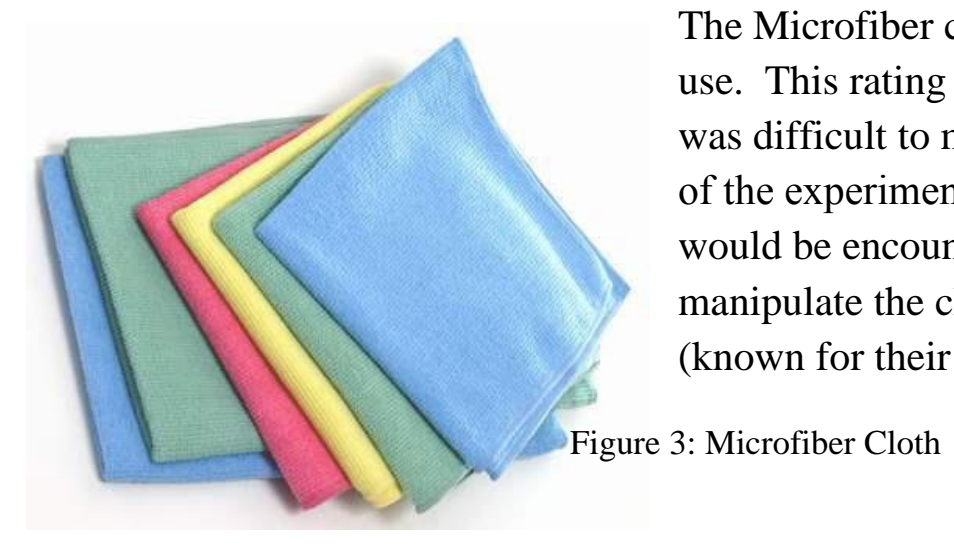


rated 7.7/10 for its effectiveness on polycarbonate and 7.6/10 for its effectiveness on orthofabric. Some mention was made of the fact that the cloth didn't collect as much dust as the wet multi-surface dust wipes, as it released some of the dust to the atmosphere of the experiment. This may or may not be relevant in an actual zero-g scenario. It should also be mentioned that the size of the cloth was no larger than 6" 6 6", thus cleaning of a large surface would be time consuming and would probably not be the cleaning tool of choice while performing time-critical operations.

\section{LCD Screen Cleaning Wipes}

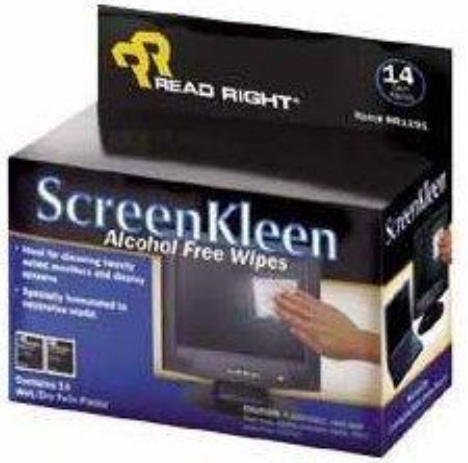

Figure 4: LCD (Dry) Screen Cleaning Wipes

The LCD cleaning wipes were rated $7.6 / 10$ for ease of use. These wipes were dry and specialized for computer and TV screens. For the same reasons that the microfiber cloth was somewhat difficult to use, the dry wipes were as well. The cloth was rated $8.3 / 10$ for its effectiveness on polycarbonate and $8.1 / 10$ for its effectiveness on orthofabric. The wipes were smaller than the microfiber cloth and thus could also require a significant amount of time to clean a large surface.

\section{Pledge Multi-Surface Dust Wipes (Wet)}

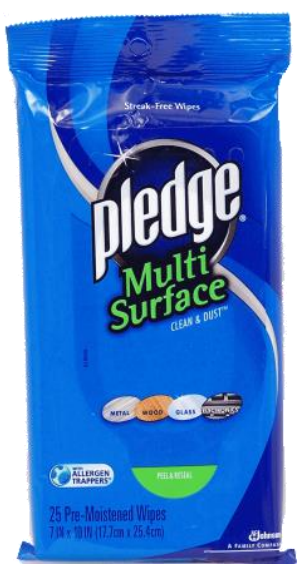

Figure 5: Pledge Multi-Surface Dust Wipes (Wet) The wet Pledge Multi-Surface wipes were rated 7.0/10 for ease of use on orthofabric and 7.2/10 for ease of use on polycarbonate. Again, the use of wipes or a cloth introduces difficulties with respect to maneuverability of the cleaning tool (cloth or wipe). The cloth was rated 8.7/10 for its effectiveness on polycarbonate and orthofabric. These wipes were the highest rated of all of the cleaning products. The moisture held in the wipes allowed regolith to be more easily collected compared to the other products. Unfortunately, if a wet dust wipe were actually used in a vacuum environment (like that of the Moon or any asteroid), the moisture would quickly dissipate from it and negate its advantage over the dry wipes.

\section{Magnetic Brush}

As previously mentioned, the brush wasn't effective at all due the humidity in the glove box. However, its ease of use was essentially rated on par with the rest of the cleaning tools, if not slightly above average. In a properly simulated space environment, a similar magnetic brush would be assumed to perform much better.

\section{Microfiber Sponge}




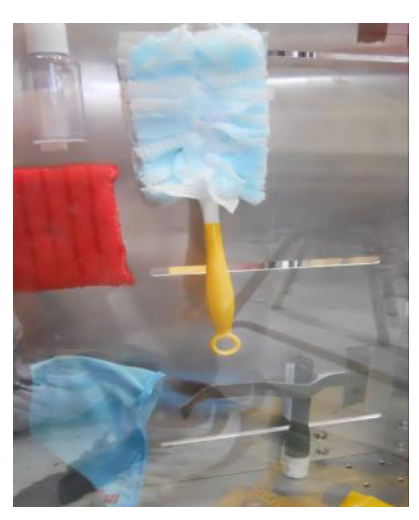

Figure 6: Microfiber Sponge

The microfiber sponge was rated 8.1/10 for ease of use on both the orthofabric and the polycarbonate. The effectiveness of the sponge was $8.0 / 10$ on the orthofabric and 7.6/10 on the polycarbonate. The effectiveness of the sponge had to do with the fact that the sponge was much thicker than the wipes and microfiber cloth, which allowed dust to penetrate the surface of the sponge and allow more dust to be collected. Additionally, the sponge was easier to scrub with when compared to the cloth and wipes. If a larger version of this sponge with the ergonomic advantages of the Swiffer Duster was tested, it may have been the best cleaner of all the products tested.

\section{Hypergravity Results}

Data was not collected during hypergravity portions of the flight.

\section{Discussion}

There was a lot learned about methodology during this experiment which should greatly benefit the second level of testing. We faced several challenges during the experiment that will lead to a better design in the future. First, the portholes in the containment box were too small and the gloves were too short to reach all areas of the box. Second, during microgravity, one hand inside the box was always being used to hold the experimenter in place. The most comfortable position always inevitably blocked the camera's view of the experiment, so data could only be gathered after a cleaning was done and the sample was revealed to the camera. Third, the camera's field of view was too small to see the two side panels, so samples on these panels did not return usable footage.

Aside from these logistical issues, the experiment was largely a success. Each commercial item tested performed better than anticipated, each one scoring above a 7.0/10 in ease of use and above a 7.6/10 in cleaning effectiveness. These results matched our 1-g results. Additionally, we now know how to modify the experiment in order to increase the effectiveness of the magnetic brush. Either a vacuum can be pulled on the containment box or nitrogen can be introduced into the box to dry the air.

\section{Outreach}

Outreach became an important factor for the team; As space advocates we look back at what influenced us to become engineers and scientists interested in space exploration, and each of us can look at a teacher, an astronaut, a space launch, or a role model who made an impact on our lives for the better. The Reduced Gravity Student Flight Opportunity Program (RGSFOP) reaches out to university students in the same way, and the UW-Madison team wanted to share this excitement with a highly motivated and intelligent group of students about to begin school at universities across the country. The objective of our outreach was to mentor a group of 12 high school students in the design and construction of their own microgravity experiment. The ultimate goal would be to bolt their experiment on the side of the UW-Madison experiment and collect data for the students to analyze and learn from. 
A team of advanced physics students from East Troy High School in Wisconsin were selected to work with us. The students researched ideas, elected team leaders, and divided into groups to accomplish all of the work for each subsystem. The UW-Madison team was able to mentor the development of the project, interact with the students, and guide them towards the ability to create an experiment for microgravity. We learned that this effort was by no means easy and a mentorship that both parties would be able to learn from.

The final result was rewarding for both the high school students and the UW-Madison team. As a diverse group of students we were able to work with minorities in science and engineering and stimulate interest not only in STEM fields but also in space exploration. Students from the high school team are pursuing microgravity experiments at the universities they will attend next year. The high school teacher with whom we had the honor to work, is looking into beginning a high school team that will apply to the HUNCH program next year (He would not have considered this otherwise). The knowledge UW-Madison students have gained as mentors as well as our impact on the high school students is irreplaceable. One high school at a time, the UW-Madison team feels that we can truly have an impact on the engineers, scientists, and explorers of the future.

\section{Conclusion}

The information gathered from FIRCE should provide a sound base for a level two test of commercial off-the-shelf products. In such a test, the glove box environment should be more representative of an actual zero-g, vacuum environment. The possibility of altering the products to test each of them at an equal ease of use should be explored. This could consist of having all of them on a common handle like that of the Swiffer Duster. An automated system utilizing the products tested in the FIRCE should also be developed. An attempt to create a prototype for this level one test was unsuccessful due to hardware failure prior to testing. This system is described in Appendix B. Overall, the results of FIRCE were positive and suggest that further research and testing should be invested to understand if there are commercial off-the-shelf products that could be adapted to help mitigate dust for future manned exploration mission

\section{Acknowledgements}

We would like to acknowledge and extend our gratitude to the following organizations and persons who have made the completion of this experiment possible:

- Wisconsin Space Grant Consortium, for their generous support in the construction of our experiment.

- Space Science and Engineering Center of UW Madison, for their generous support and commitment to our team throughout the entire process.

- Professor Bonazza, for his encouragement and guidance.

- Dr. Elder, for his understanding and assistance.

- NASA's Reduced Gravity Education Flight Program and all of its employees, for giving us this amazing opportunity and experience.

- Robert Trevino, our mentor, without him none of this would have been possible. Without the support and effort of these organizations and individuals, the completion of the experiment would not have been possible 


\section{References}

[1] Cadogan, D., \& Ferl, J. (2007). Dust Mitigation Solutions for Lunar and Mars Surface Systems.

[2] Soil Science Society of America (2008, September 24). NASA's Dirty Secret: Moon Dust. ScienceDaily. Retrieved February 18, 2012, from http://www.sciencedaily.com /releases/2008/09/080924191552.html 